05

\title{
Влияние параметров стабилизирующего слоя 2G ВTCП лент и особенностей теплоотвода на форму BAX в резистивном состоянии и на критическое напряжение, вызывающее необратимые изменения сверхпроводящих свойств
}

\author{
(C) В.А. Мальгинов, ${ }^{1}$ А.В. Мальгинов, ${ }^{2}$ Д.А. Горбунова ${ }^{3}$ \\ ${ }^{1}$ Физический институт им. П.Н. Лебедева РАН, 119991 Москва, Россия \\ ${ }^{2}$ Многофункциональный общий центр обслуживания Госкорпорации „Росатом“ акционерное общество „Гринатом“, \\ 115230 Москва, Россия \\ ${ }^{3} \mathrm{OОO} \mathrm{„С-Инновации“,}$ \\ 117246 Москва, Россия \\ e-mail: malginov@sci.lebedev.ru
}

(Поступило в Редакцию 10 июля 2017 г. В окончательной редакции 17 октября 2017 г.)

Экспериментально определен реальный вид резистивного участка вольт-амперной характеристики (BAX) ВТСП ленты на переменном токе. Установлено, что ВАХ определяется как электрическими свойствами сверхпроводящего слоя, так и термомеханическими свойствами слоев в структуре ленты. Найдены особенности поведения BAX в разных диапазонах токов. Показано, что ниже критического тока малые тепловыделения в стабилизаторе и сверхпроводящем слое не влияют на вид ВАХ. При превышении критического тока совместные тепловыделения в стабилизирующем и сверхпроводящем слоях вызывают повышение температуры и сопротивления ВТСП ленты в резистивном состоянии.

Обнаружено, что существует критический уровень толщины стабилизирующего слоя. Ниже этого уровня основной ток в резистивном состоянии течет по ВТСП слою, и при малом его перегреве образец переходит в нормальное состояние из-за тепловой нестабильности теплоотвода в жидкий азот. Для лент с большей толщиной медного слоя ток в резистивном состоянии перераспределяется между стабилизирующим и ВТСП слоями и переход в нормальное состояние происходит вблизи критической температуры сверхпроводника при нулевом токе. Установлена зависимость критических электрических напряжений, которые характеризуют разную степень разрушения ВТСП лент от толщины медного слоя. Обнаружено, что при подаче скачкообразного напряжения разрушение происходит при более высоких напряжениях.

DOI: 10.21883/JTF.2018.05.45902.2419

\section{Введение}

Исследование и анализ процессов, происходящих в композитных сверхпроводниковых материалах при протекании токов выше критического, служат основой для создания электротехнических устройств, удовлетворяющих требованиям электрических сетей по надежности, термической и электродинамической стойкости [1-3]. Изучение механизмов перехода ВТСП лент из резистивного в нормальное состояние позволяют найти уровень устойчивости ленты к перегреву и определить критическое напряжения, которое приводит к необратимой потере сверхпроводящих свойств [4]. Исследование условий формирования реальной ВАХ в резистивном состоянии сверхпроводников является одной из основных задач, возникающих при разработке безынерционных термически стойких ВТСП токоограничивающих устройств [5,6]. В простейшем виде ВАХ описывается известным степенным уравнением [2]. Теоретическое изучение процессов формирования макроскопических состояний сверхпроводящей ленты показывает отклонение от этого уравнения и существование характерных значений напряженности электрического поля, зависящих от условий охлаждения и свойств сверхпроводника и стабилизирующей матрицы, которые лежат в основе теплоэлектродинамических механизмов, влияющих на крутизну нарастания вольт-амперных характеристик технических сверхпроводников [7]. В настоящее время для ВТСП композитных материалов вопрос о влиянии этих факторов на вид ВАХ еще не имеет однозначного решения. А для переменного тока (особенно актуального для практических применений) появляется еще один фактор, влияющий на вид начальной стадии ВАХ. Это вихревые тепловыделения в стабилизирующих слоях ВТСП ленты. Было показано [3], что для криостатической стабилизации сверхпроводящего состояния и повышения максимального тока резистивной ветви ВАХ (ток теплового срыва) низкотемпературных композитных сверхпроводников необходимо увеличивать относительную концентрацию нормального металла в них. Однако в [8] для 2G ВТСП ленточных проводов на основании анализа теоретической модели утверждается, что возможность повышения максимально допустимого тока с помощью стабилизирующего покрытия весьма ограничена (до 20\%), при этом экспериментальные данные для определенных стабилизированных лент показывают уровень этого повышения около 100\% [9]. Таким образом, остается открытым вопрос об экспериментальном 
изучении влияния количества стабилизатора в ВТСП ленте на уровень тока теплового срыва.

При превышении током в ВТСП ленте тока теплового срыва баланс между тепловыделениями и теплоотводом нарушается и происходит значительный нагрев ленты (тепловой „срыв“ или квенч). Ввиду наличия продольной неоднородности ее свойств разогрев имеет локальный характер, что особенно выражено в 2G ВТСП материалах со сложной структурой [10]. Локальные тепловыделения приводят к перегреву выше $500 \mathrm{~K}$ [9] и необратимой деградации сверхпроводящих свойств.

Таким образом, для понимания механизмов, способствующих повышению тока теплового срыва, для нахождения причин, вызывающих необратимую потерю сверхпроводимости в ВТСП токонесущих элементах с различной толщиной стабилизатора при токовой и тепловой перегрузке на переменном токе, необходимо детальное экспериментальное исследование процесса перехода как из сверхпроводящего состояния в резистивное, так и из резистивного в нормальное. Этому и посвящена настоящая работа.

\section{Влияние параметров стабилизирующего слоя 2G ВТСП лент на вид ВАХ В резистивном состоянии}

Для экспериментального исследования электрических, тепловых и механических процессов в композитных ВТСП лентах на всех стадиях перехода их в нормальное состояние были использованы образцы фирмы СуперОкс с одинаковой структурой ВТСП слоя и различной (от 0 до $40 \mu \mathrm{m}$ ) толщиной $d$ медного стабилизирующего слоя. Поскольку медные $(\mathrm{Cu})$ слои расположены с двух сторон ленты, а толщина слоя

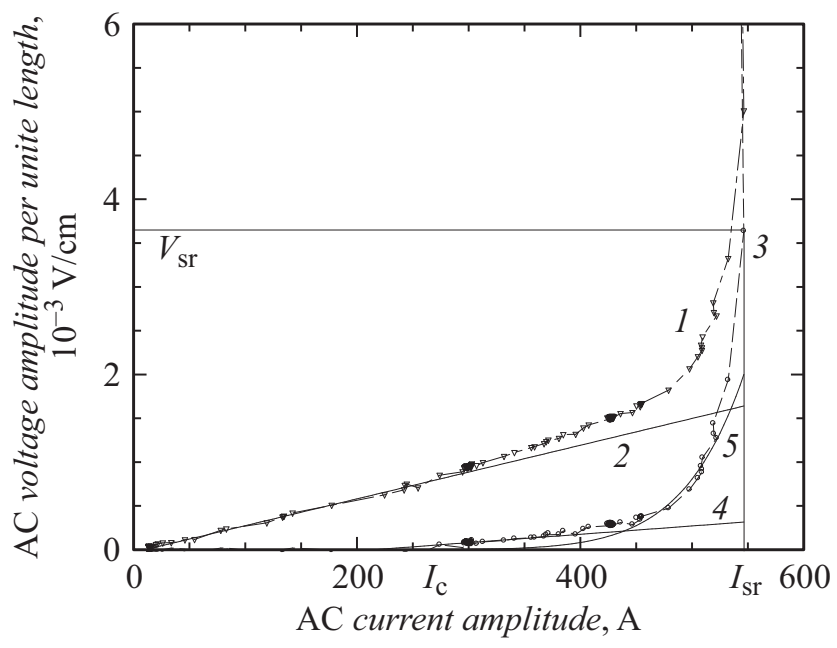

Рис. 1. Амплитудные BAX на переменном токе образца с толщиной медного стабилизатора $9 \mu \mathrm{m} .1-\mathrm{BAX}$ с индуктивной составляющей, $2-\mathrm{BAX}$ индуктивной части, 3 - ВАХ на резистивном сопротивлении образца, 4 $V / L=10^{-6}(I-260)+40 \cdot 10^{-6}, 5-V / L=10^{-6}(I / 260)^{10.2}$. серебра (Ag) равна $2 \mu \mathrm{m}$, суммарная толщина $\mathrm{Cu} / \mathrm{Ag}$ стабилизатора равна $2 d+2 \mu \mathrm{m}$. Слой серебра наносился на ВТСП ленту с обеих сторон с применением метода магнетронного распыления металлического серебра. Толщина слоя контролировалась при помощи электронной микроскопии. Равномерный слой меди наносился на ВТСП ленту со всех сторон электрохимическим методом с применением сернокислого электролита при комнатной температуре на специально сконструированном оборудовании. Толщина слоя меди контролировалась за счет варьирования скорости движения ленты и силы пропускаемого тока. Различные стадии процесса получения ВТСП провода и их особенности более подробно описаны в публикациях $[11,12]$.

Для проведения измерений была применена методика на переменном токе частотой $50 \mathrm{~Hz}$ [4]. Перевод в нормальное состояние осуществлялся посредством импульсов переменного напряжения двух типов : 1-й тип - напряжение с плавным повышением амплитуды в течение 8-10 s. 2-й тип - скачкообразное повышение амплитуды напряжения, когда в течение первых $0.1 \mathrm{~s}$ напряжение повышается до значения более чем $80 \%$ от максимального значения и затем поддерживается на максимальном уровне в течение 8-10s.

Характерное поведение амплитудных ВАХ при плавном повышении напряжения представлено на рис. 1 для образца с толщиной медного слоя в $9 \mu \mathrm{m}$. Кривая 1 показывает вид полного сигнала на образце. До критического тока $I_{c}$ (для рассматриваемых образцов $\left.I_{c} \approx 260 \mathrm{~A}\right)$ ВАХ имеет линейную зависимость (кривая 2), которая связана с индуктивным сопротивлением прямолинейных ленточных проводников [13]. После вычитания индуктивной и контактной составляющих получаем кривую 3, которая характеризует поведение активного сопротивления образца в сверхпроводящем, резистивном и нормальном состояниях.

Ниже критического тока малые тепловыделения в стабилизаторе и ВТСП слое не влияют на вид ВАХ. Для амплитуды тока $I$ в диапазоне $(1-1.5) I_{c}$ напряженность в резистивном состоянии $V / L(V-$ напряжение на образце, $L$ - его длина) хорошо апроксимируется линейной функцией (кривая 4):

$$
V / L=a\left(I-I_{c}\right)+b,
$$

где $a$ и $b-$ постоянные коэффициенты.

При токе от $1.7 I_{c}$ до 2.0I ВАХ ВТСП ленты хорошо описывается применяемой для сверхпроводников [2], степенной функцией (кривая 5):

$$
V / L=10^{-6}\left(I / I_{c}\right)^{n},
$$

$n$ - показатель степени.

Размерности величин, входящих в формулы (1) и (2), имеют следующие значения: напряжение $-V, L-\mathrm{cm}$, $I-\mathrm{A}, a-\Omega / \mathrm{cm}, b-\mathrm{V} / \mathrm{cm}$.

Значительное отличие напряженности ленты при токах $(1-1.5) I_{c}$ (кривая 4) от напряженности сверхпроводника на постоянном токе (кривая 5), связано с 


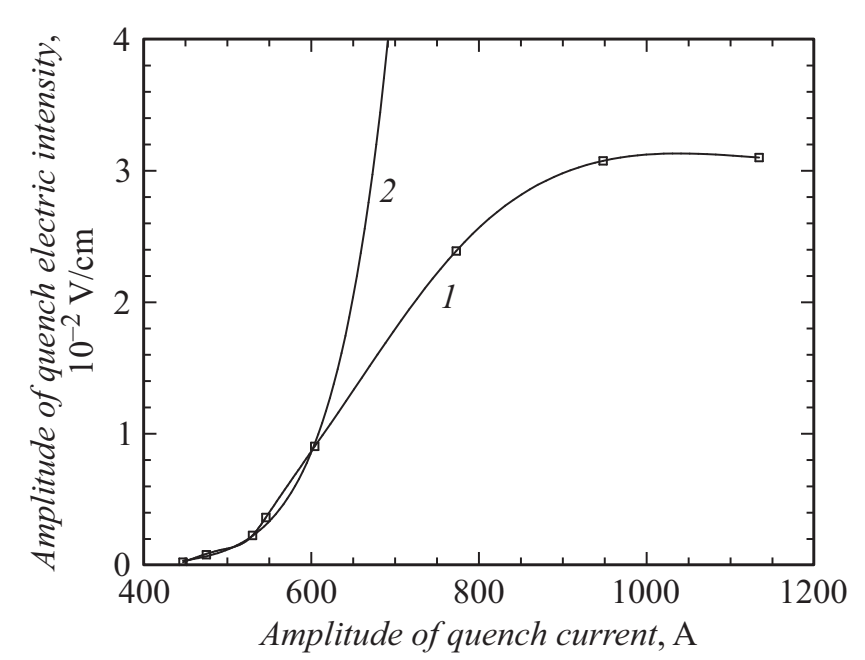

Рис. 2. Взаимосвязь максимальной напряженности и тока резистивного состояния в образцах с различной толщиной стабилизирующего слоя: 1 - экспериментальные данные, 2 $V_{s r} / L=10^{-6}(\text { Isr } / 260)^{10.8}$.

тем, что на переменном токе, за счет индуктивного напряжения (кривая 2), происходят тепловыделения в $\mathrm{Cu} / \mathrm{Ag}$ стабилизирующих слоях, которые экранируют теплоотвод от ВТСП слоя в омывающий ленту хладагент. Этот эффект вызывает повышение температуры и сопротивления ВТСП слоя, что приводит к увеличению крутизны ВАХ. При превышении тока $1.5 I_{c}$ повышение температуры ВТСП слоя уже таково, что малые тепловыделения в стабилизирующем слое не могут ограничивать теплоотвод от ВТСП слоя в хладагент. В этом случае ВАХ ленты выходит на уровень, характерный для сверхпроводников (кривая 5).

По визуальному наблюдению характера кипения азота на поверхности ВТСП ленты и виду ВАХ (рис. 1) можно определить, какой фазовой стадии соответствует каждая ветвь BAX. До критического тока $I_{c}$ образец существует в сверхпроводящем состоянии. От $I_{c}$ до тока теплового перехода $I_{s r}$ образец находится в резистивном состоянии, тепловыделения и кипение азота происходят по всей длине образца. После превышения $I_{s r}$ и напряжения теплового срыва $V_{s r}$, часть образца переходит в нормальное состояние.

Предельные значения $I_{s r}$ и $V_{s r}$ тока и напряженности монотонно растут по мере увеличения толщины стабилизатора. Их соотношение для разных толщин $\mathrm{Cu}$ стабилизатора представлено на рис. 2.

Из рисунка видно, что если ток в резистивном состоянии не превышает $2 I_{c}$, то $\mathrm{BAX}$ максимальных значений описывается степенной функцией, характерной для сверхпроводников. При увеличении максимального тока поведение ВАХ существенно отклоняется от этой зависимости. Причину этого легко понять из анализа поведения сопротивления различных слоев и ленты в целом при различной толщине медного стабилизатора рис. 3.
На этом рисунке кривая 1 показывает сопротивление $V_{s r} / I_{s r}$ всей ленты, а кривая 2 - суммарное сопротивление $\mathrm{Cu} / \mathrm{Ag}$-слоев. Поскольку в ленте $\mathrm{Cu} / \mathrm{Ag}$-слои и ВТСП слой соединены параллельно, а слой подложки из нержавеющей стали для всех толщин стабилизирующих слоев имеет сопротивление более чем на порядок большее сопротивления этих слоев, из кривых 1 и 2 легко получить зависимость максимального сопротивления резистивного состояния ВТСП слоя (кривая 3). Таким образом, из рисунка видно, что при толщине $\mathrm{Cu}$-слоя менее $13 \mu \mathrm{m}$ сопротивление ленты определяется резистивным сопротивлением ВТСП слоя и бо́льшая часть тока течет по ВТСП слою. При толщине $\mathrm{Cu}-$ слоя более $27 \mu \mathrm{m}$ ток при значениях близких к $I_{s r}$ практически весь течет по $\mathrm{Cu} / \mathrm{Ag}$ стабилизирующим слоям. В диапазоне толщин $\mathrm{Cu}-$ слоя от 13 до $27 \mu \mathrm{m}$ сопротивления слоев имеют один порядок, и общий ток ленты перераспределяется между слоями обратно пропорционально их сопротивлению.

Вид BAX для двух разных значений толщин $\mathrm{Cu}-$-лоя во всем диапазоне токов представлен на рис. 4. Для всех значений толщин $\mathrm{Cu}$ для токов, не превышающих $2 I_{c}$, вид ВАХ определяется выражениями (1) и (2) со следующими значениями параметров: $a \approx 10^{-6} \Omega / \mathrm{cm}$, $b \approx 40 \cdot 10^{-6} \mathrm{~V} / \mathrm{cm}, n \approx 10$. При токах выше значений $2 I_{c}$ поведение $\mathrm{BAX}$ для разных толщин $\mathrm{Cu}$-слоя существенно отличается - рис. 4. Из характера поведения ВАХ при большой толщине слоя Си (кривая 2, $d=40 \mu \mathrm{m})$ видно, что при тепловыделениях в ленте около $2 \mathrm{~W} / \mathrm{cm}^{2}$ в течение $0.3-0.5 \mathrm{~s}$ теряется монотонность BAХ и происходит всплеск сопротивления ленты. Этот эффект связан с тем, что при перегреве ленты в $1 \mathrm{~K}$ происходит смена конвективного режима теплоотдачи на пузырьковый режим кипения [14]. При смене режимов

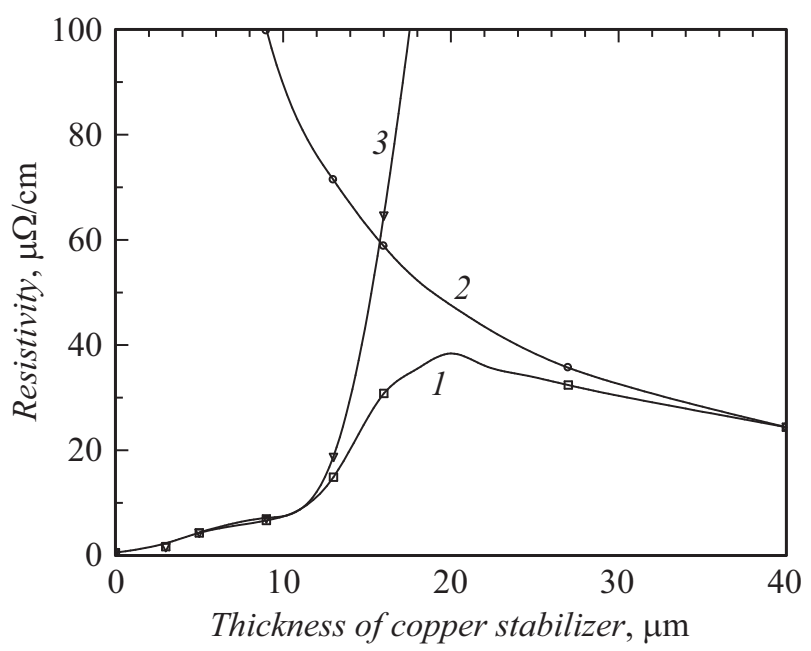

Рис. 3. Сопротивление различных слоев ВТСП ленты в образцах с различной толщиной стабилизирующего слоя: 1 максимальное сопротивление ленты в резистивном состоянии, экспериментальные данные, 2 - сопротивление $\mathrm{Cu} / \mathrm{Ag}$-слоев, 3 - максимальное сопротивление резистивного состояния ВТСП слоя. 


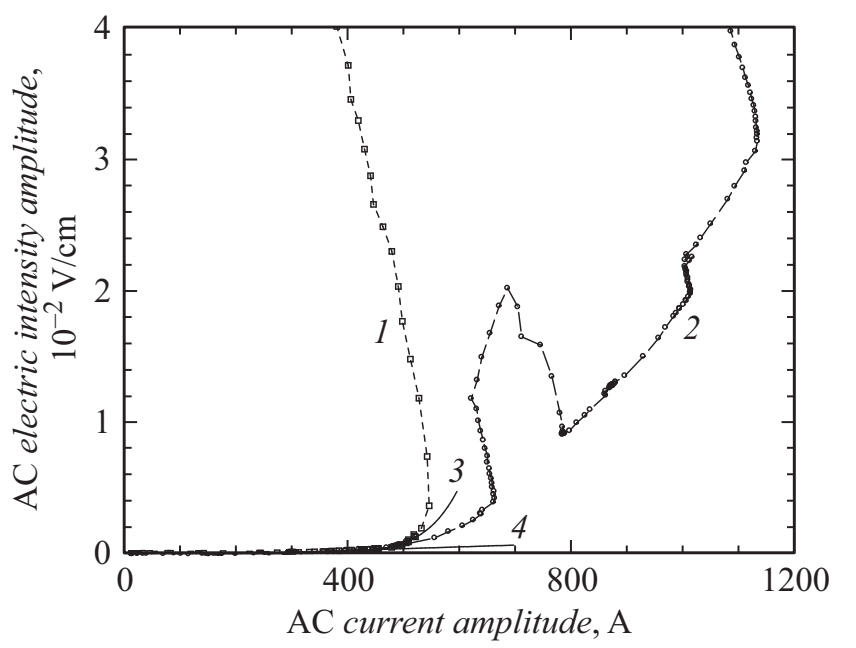

Рис. 4. Амплитудные BAX на переменном токе образцов с разной толщиной медного стабилизатора: 1 - толщина $9 \mu \mathrm{m}, 2-$ толщина $40 \mu \mathrm{m}, 3-V / L=10^{-6}(I / 260)^{10.2}, 4-$ $V / L=10^{-6}(I-260)+40 \cdot 10^{-6}$.

резко падает уровень теплового потока с поверхности ВТСП ленты в жидкий азот. Это приводит к тому, что происходит более резкий разогрев ленты в квазиадиабатическом режиме. Если основной ток течет по ВТСП слою и запас у ленты до критической температуры мал, то в этот момент происходит ее переход в нормальное состояние. Как видно из рис. 1-4 такое поведение характерно для ВТСП лент с толщиной медного стабилизатора не более $13 \mu \mathrm{m}$. Для лент с большой толщиной Сислоя (рис. 4, кривая 2) часть тока начинает перетекать из ВТСП слоя в $\mathrm{Cu} / \mathrm{Ag}$-стабилизатор, при этом повышается температурный запас до перехода в нормальное состояние. В этой ситуации при плотности тепловыделений около $2 \mathrm{~W} / \mathrm{cm}^{2}$ происходит скачок сопротивления, а переход в нормальное состояние происходит вблизи критической температуры сверхпроводника при нулевом токе (около 92 К) и при теплоотдаче с поверхности ленты, характерной для пузырькового кипения.

\section{Влияние параметров стабилизирующего слоя 2G ВТСП лент на критическое напряжение, вызывающее необратимые изменения сверхпроводящих свойств}

При переходе в нормальное состояние нормальная зона сосредоточена в небольшой области. Длина этого нормального домена составляет несколько сантиметров [4] и она слабо зависит от длины всего образца. В этой области существует концентрация электрического и механического напряжений. Для того чтобы определить минимальное значение напряжения $V_{r}$, при котором начинается изменение резистивной области $\mathrm{BAX}$, необходимо, чтобы $V_{s r}$ (а значит и длина образца) были меньше критической величины $V_{r}$. Поэтому при определении критических напряжений, вызывающих изменение сверхпроводящих свойств ВТСП материала, использовались образцы длиной $7 \mathrm{~cm}$, а для более точного определения параметров только резистивного состояния $\left(V_{s r}\right.$ и $\left.I_{s r}\right)$ использовались образцы длиной $24 \mathrm{~cm}$.

Изменение вида ВАХ при увеличении подаваемого на образец напряжения представлено на рис. 5. При подаче максимального напряжения до критического значения $V_{r}$ характер ВАХ меняется мало и имеет вид кривой 1 . При превышении напряжения $V_{r}$ происходит необратимое увеличение крутизны ВАХ в начальной стадии резистивного состояния (кривая 2). При дальнейшем повышении напряжения и превышении следуюшего критического значения $V_{k}$ начинается необратимая деградация критического тока. При напряжении, равном $V_{s}$, критический ток снижается до нуля (кривая 3). Поскольку электрические, тепловые и механические нагрузки происходят в нормальном домене, то и изменения в $\mathrm{BAX}$ связаны с изменениями в этой области. Так как при напряжении $V_{k}$ (кривая 2) еще не происходит изменения сверхпроводящих свойств ВТСП слоя, изменение наклона начальной стадии (в диапазоне токов $\left.(1-1.5) I_{c}\right)$ ВАХ в 4 раза (кривая 5) связано с повышением температуры в резистивном состоянии за счет увеличения теплового сопротивления между ВТСП слоем и хладагентом в той области, где возникал нормальный домен. Это означает, что в этой области в то время, когда образец находился в нормальном состоянии, произошло частичное отслоение слоя серебра от ВТСП слоя. Существенные изменения в $\mathrm{BAX}$ произошли и при токах выше $1.5 I_{c}$. Теперь скачки сопротивления, вызванные сменой режима теплоотдачи, уже не вызывают перехода в нормальное состояние (кривая 2). Для этой части образца повышается устойчивость к тепловым флуктуациям и значительно увеличивается предельное напряжение резистивного состояния $V_{s r}$.

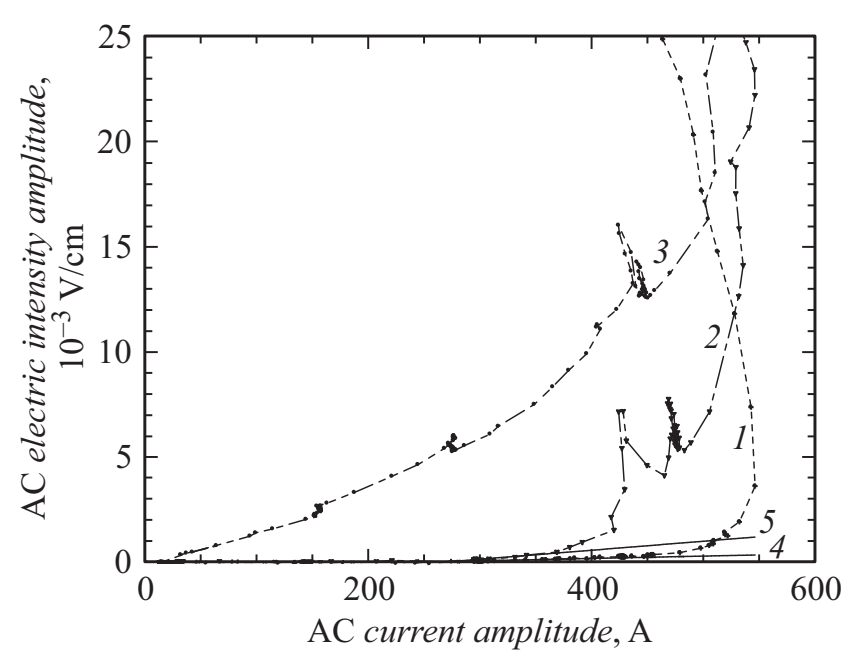

Рис. 5. Изменение ВАХ при изменении максимального напряжения на образце с толщиной медного стабилизатора $9 \mu \mathrm{m}: 1-V_{r}=0.49 \mathrm{~V}, 2-V_{k}=0.85 \mathrm{~V}, 3-$ $V_{s}=0.95 \mathrm{~V}, \quad 4-V / L=10^{-6}(I-260)+40 \cdot 10^{-6}, 5-$ $V / L=4.3 \cdot 10^{-6}(I-260)$. 


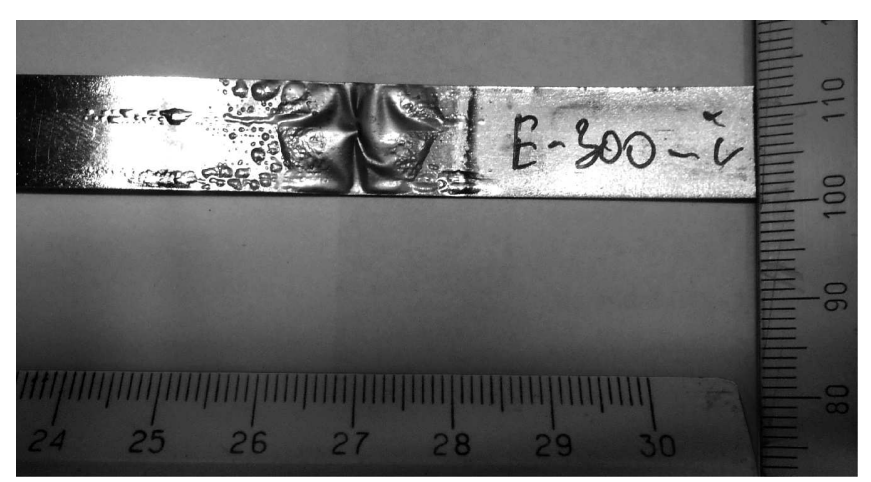

Рис. 6. Внешний вид ВТСП ленты с толщиной стабилизатора $27 \mu \mathrm{m}$ после ее перехода в нормальное состояние при напряжении $1.9 \mathrm{~V}$.

Из этого следует, что отслоение слоя серебра, кроме ухудшения теплоотдачи, выполняет и другую функцию: убираются механически напряженные места, возникшие между ВТСП и Ag-слоями при охлаждении ленты до температуры жидкого азота.

Типичный пример характера разрушения ВТСП ленты при подаче напряжения выше $V_{s}$ показан на рис. 6 для ленты с толщиной стабилизатора $27 \mu \mathrm{m}$. Поскольку известно, что температура в нормальном домене уменьшается от центра к периферии [4] и что максимальная температура монотонно повышается с напряжением [9], из рис. 6 можно оценить характер деформации стабилизирующего слоя при разной степени превышения критического напряжения $V_{s}$. Из рисунка видно, что при напряжении $1.9 \mathrm{~V}$ отслоение стабилизатора идет по всей ширине ленты. По мере снижения температуры и напряжения идет частичное отслоение с характерным размером областей деформации в $1-2 \mathrm{~mm}$. Такой характер деформации наблюдается визуально для всех образцов.

Таким образом, вид ВАХ ВТСП ленты и характер деформации ее структуры при переходе в нормальное состояние определяется не только электрическими параметрами $\mathrm{Cu}, \mathrm{Ag}$ и ВТСП слоев, но и зависит от их термомеханических свойств. Результаты, приведенные на рис. 5 и 6 , легко понять при анализе термомеханических процессов в ВТСП ленте. Поскольку коэффициенты теплового расширения слоев ВТСП ленты сильно отличаются (в частности, у слоя стабилизатора и сверхпроводящего слоя отличие составляет 2-3 раза), отклонение от температуры, при которой на ленту наносился слой стабилизатора, приводит к механическому напряжению на границах ВТСП слоя с соседними слоями. При использовании ВТСП лент они подвергаются значительным термомеханическим деформациям в двух случаях: при снижении температуры всей ленты более чем на $200 \mathrm{~K}$ при охлаждении ее до температуры жидкого азота и при переходе части ленты в нормальное состояние, когда температура участка с нормальной зоной может превышать 400-600 K [9].
При захолаживании образца до температуры жидкого азота слой $\mathrm{Cu} / \mathrm{Ag}$ стабилизатора имеет более сильную деформацию сжатия, чем ВТСП слой. За счет этого между слоями стабилизатора и сверхпроводника возникает деформация сдвига, которая создает механически напряженные места, в которых снижается устойчивость ленты к тепловым возмущениям.

Во время перехода в нормальное состояние происходит разогрев различных слоев и до комнатной температуры деформация сжатия и сдвига уменьшаются. При дальнейшем повышении температуры в слоях происходит деформация расширения, а на границе стабилизирующего и сверхпроводящего слоев деформация сдвига будет уже другого знака. Принципиальное отличие при подаче на образец плавного и резкого напряжений заключается в том, что при плавном изменении напряжения одновременно с разогревом и расширением $\mathrm{Cu}$

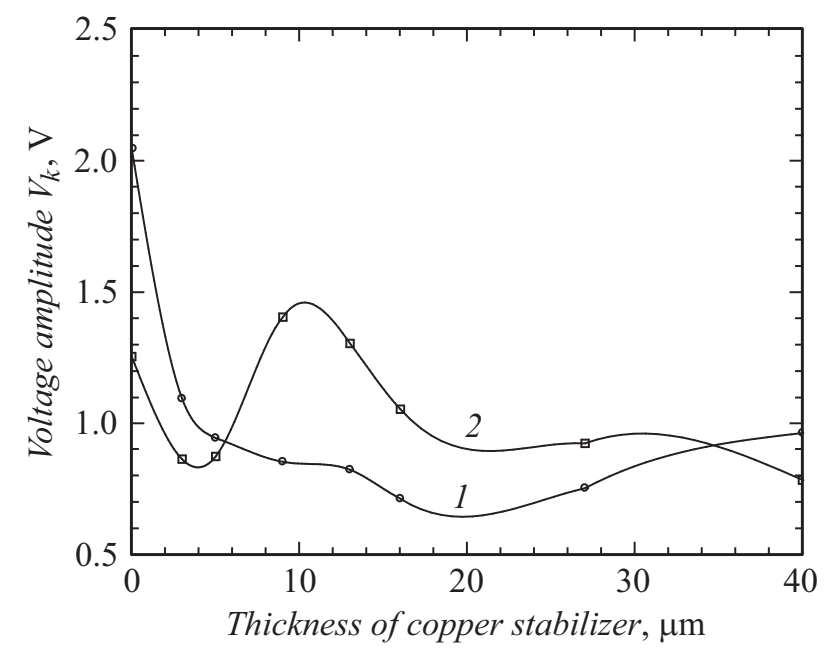

Рис. 7. Зависимость напряжения начала деградации критического тока от толщины медного слоя: 1 - плавная подача напряжения, 2 - подача напряжения скачком.

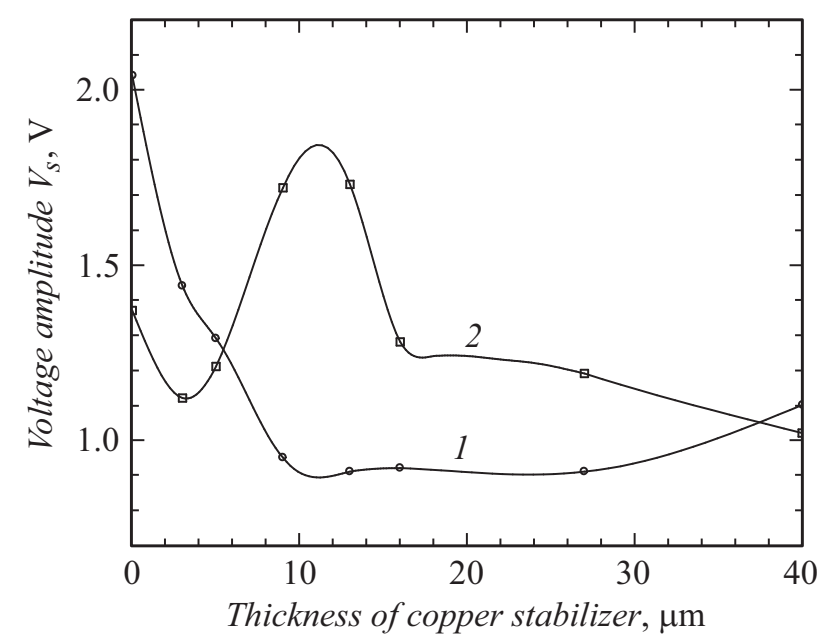

Рис. 8. Зависимость напряжения полного разрушения сверхпроводимости от толщины медного слоя: 1 - плавная подача напряжения, 2 - подача напряжения скачком. 
стабилизатора разогревается и расширяется ВТСП слой. Когда происходит превышение комнатной температуры, в ВТСП слое возникает деформация растяжения, а на границе с Ag и буферным слоем - деформация сдвига. При подаче на образцы скачкообразного напряжения в начальный момент только слои $\mathrm{Ag}$ и $\mathrm{Cu}$ имеют повышенную температуру, а остальные слои имеют более низкую температуру, близкую к температуре азотной ванны. Ускоренный разогрев и расширение стабилизирующего слоя приводит к более сильной и быстрой деформации сдвига между Ag и ВТСП слоями и к отслоению $\mathrm{Ag}$ в наиболее напряженных местах. Ликвидация наиболее слабых мест и является причиной повышения устойчивости ВТСП лент при подаче скачкообразного электрического напряжения малой амплитуды. Это видно из поведения параметров $V_{k}$ и $V_{s}$, которые характеризуют разную степень разрушения ВТСП лент, в зависимости от толщины медного слоя при разных режимах подачи внешнего напряжения (рис. 7,8). Из рисунков также видно, что деградация сверхпроводящих свойств в установившемся режиме происходит при напряжении на нормальной зоне в $0.5-1.0 \mathrm{~V}$.

Слабая зависимость критических напряжений от толщины стабилизатора во время плавного изменения напряжения при толщине стабилизирующего слоя, значительно превышающей толщину ВТСП слоя, указывает на то, что в этом случае причиной разрушения ВТСП слоя является не толщина стабилизирующего слоя, а его деформация вследствие температуры, которая достигается во время перехода ленты в нормальное состояние.

\section{Выводы}

Экспериментально установлено, что резистивный участок ВАХ ВТСП ленты определяется как электрическими свойствами сверхпроводящего слоя, так и термомеханическими свойствами слоев в структуре ленты. Найдены особенности поведения ВАХ в разных диапазонах токов. Показано, что ниже критического тока малые тепловыделения в стабилизаторе и сверхпроводящем слое не влияют на вид ВАХ. Обнаружено, что при токах $(1-1.5) I_{c}\left(I_{c}-\right.$ критический ток) напряженность значительно превышает значения для сверхпроводника на постоянном токе, что связано с тем, что за счет индуктивного напряжения происходят тепловыделения в $\mathrm{Cu} / \mathrm{Ag}$ стабилизирующих слоях, которые экранируют теплоотвод от ВТСП слоя и вызывают повышение его температуры и сопротивления. При токе от 1.5 до $2.0 I_{c}$ ВАХ ВТСП ленты хорошо описывается применяемой для сверхпроводников на постоянном токе степенной функцией с показателем около 10.

Обнаружено, что существует критический уровень толщины стабилизатора (около $13 \mu \mathrm{m}$ ), в котором меняется характер ВАХ перед переходом образца в нормальное состояние. Ниже этого уровня основной ток в резистивном состоянии течет по ВТСП слою и при малом его перегреве образец переходит в нормальное состояние из-за тепловой нестабильности теплоотвода в жидкий азот. Для лент с большей толщиной медного слоя ток в резистивном состоянии перераспределяется между стабилизирующим и ВТСП слоями и переход в нормальное состояние происходит вблизи критической температуры сверхпроводника при нулевом токе и при теплоотдаче с поверхности ленты, характерной для пузырькового кипения.

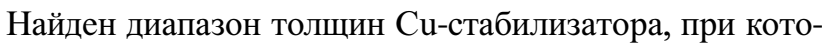
ром сопротивление в резистивном состоянии достигает наибольших значений. Установлено, что в резистивном состоянии сопротивление равномерно распределено по длине образца, а деградация сверхпроводящих свойств происходит во время перехода в нормальное состояние при амплитуде установившегося напряжения на нормальном участке в $0.5-1.0 \mathrm{~V}$.

Установлена зависимость критических электрических напряжений, которые характеризуют разную степень разрушения ВТСП лент, от толщины медного слоя при разных режимах подачи внешнего напряжения. Обнаружено, что при подаче скачкообразного напряжения при толщинах стабилизатора выше $5 \mu \mathrm{m}$ разрушение начинается при более высоких напряжениях.

Авторы благодарят генерального директора фирмы СуперОкс С.В. Самойленкова за предоставление для исследований серии ВТСП образцов с переменной толщиной стабилизатора. Особую благодарность авторы выражают B.M. Пудалову за постоянную поддержку выбранного направления изучения ВТСП материалов и за ряд ценных замечаний при подготовке данной публикации.

Работа выполнена на оборудовании ЦКП ФИАН, при финансовой поддержке Программы фундаментальных исследований президиума РАН „Фундаментальные проблемы высокотемпературной сверхпроводимости“ и РФФИ в рамках научного проекта № 17-29-10003.

\section{Список литературы}

[1] Альтов В.А., Зенкевич В.Б., Кремлев М.Г., Сычев В.В. Стабилизация сверхпроводящих магнитных систем. М.: Энергоатомиздат, 1984. $312 \mathrm{c.}$

[2] Уилсон М. Сверхпроводящие магниты. М.: Мир, 1985. $407 \mathrm{c}$.

[3] Гуревич А.Вл., Мини Р.Г., Рахманов А.А. Физика композитных сверхпроводников. М.: Наука, 1987. 240 с.

[4] Мальгинов А.В., Кунщевич А.Ю., Мальгинов В.А., Флейшман Л.С. // ЖЭТФ. 2013. Т. 144. Вып. 6. С. 1225-1238.

[5] Флейшман Л.С., Мальгинов В.А., Мальгинов А.В. // Известия РАН. Энергетика. 2010. № 5. С. 61-67.

[6] Fleishman L.S., Volkov E.P., Malginov V.A. et al. // IEEE Trans. Appl. Supercond. 2011. Vol. 21. N 3. P. 1263-1266.

[7] Романовский В.Р. / ЖТФ. 2017. Т. 87. Вып. 1. С. 49-58.

[8] Рахманов А.Л., Иванов С.С. // Электричество. 2015. N 10. C. $38-47$. 
[9] Мальгинов В.А., Мальгинов А.В., Флейшман Л.С., Ракитин А.С. // ЖТФ. 2017. Т. 87. Вып. 10. С. 1509-1517.

[10] Choi J.H., Choi Y.H., Kang D.-H. et al. // IEEE Trans. Appl. Supercond. 2015. Vol. 25. N 3. P. 6600505.

[11] Samoilenkov S., Molodyk A., Lee S. et al. // Supercond. Sci. Technol. 2016. Vol. 29. N 2. P. 024001.

[12] Lee S., Petrykin V., Molodyk A. et al. // Supercond. Sci. Technol. 2014. Vol. 27. N 4. P. 044022.

[13] Калантаров П.Л., Цейтлин Л.А. Расчет индуктивностей. Л.: Энергоатомиздат, 1986. С. 102.

[14] Григорьев В.А., Павлов Ю.М., Аметистов Е.В. Кипение криогенных жидкостей. М.: Энергия, 1977. С. 288. 